\title{
Cisplatin augments cytotoxic T-lymphocyte-mediated antitumor immunity in poorly immunogenic murine lung cancer
}

\author{
Robert E. Merritt, $\mathrm{MD}^{\mathrm{a}}$ \\ Ali Mahtabifard, $\mathrm{MD}^{\mathrm{a}}$ \\ Reiko E. Yamada, $\mathrm{BA}^{\mathrm{a}}$ \\ Ronald G. Crystal, MD ${ }^{\mathrm{b}, \mathrm{c}}$ \\ Robert J. Korst, MD ${ }^{\mathrm{a}, \mathrm{c}}$
}

From the Department of Cardiothoracic Surgery $^{\mathrm{a}}$ and Division of Pulmonary and Critical Care Medicine, Department of Medicine, ${ }^{\mathrm{b}}$ and Department of Genetic Medicine, Weill Medical College of Cornell University, ${ }^{\mathrm{c}}$ New York, NY.

These studies were supported, in part, by the Will Rogers Memorial Fund, Los Angeles, Calif, and Gen Vec, Inc, Gaithersburg, Md, and grants from The Thoracic Surgery Foundation for Research and Education (R.J.K.) and The American Lung Association (R.J.K.).

Received for publication Nov 7, 2002; revisions requested March 11, 2003; revisions received March 18, 2003; accepted for publication March 27, 2003

Address for reprints: Robert J. Korst, MD, Department of Cardiothoracic Surgery, M 404, Weill Medical College of Cornell University, 525 E. 68th St, New York, NY 10021 (E-mail: rjk2002@med.cornell.edu).

J Thorac Cardiovasc Surg 2003;126: 1609-17

Copyright (C) 2003 by The American Association for Thoracic Surgery

0022-5223/2003\$30.00+0

doi:10.1016/S0022-5223(03)00707-4
Objective: Many tumors are poorly immunogenic and resistant to cytotoxic Tlymphocyte-mediated cell lysis. Because cisplatin has been demonstrated to increase tumor cell Fas receptor expression, we hypothesized that cisplatin will enhance cytotoxic T-lymphocyte tumor cell killing and augment the antitumor effect of an active immunotherapy strategy in a poorly immunogenic murine lung cancer model.

Methods: Lewis lung carcinoma cells were exposed to cisplatin in vitro, and Fas receptor expression and apoptosis in response to an agonistic anti-Fas antibody were quantified using flow cytometry. Wild-type and Fas ligand-deficient mice bearing Lewis lung carcinoma flank tumors were then treated with intraperitoneal cisplatin as well as an intratumoral injection of an adenovirus gene transfer vector encoding CD40 ligand. End points included tumor size, animal survival, and Fas expression (determined using immunofluorescence). Cytotoxicity assays were performed using splenocytes from adenovirus gene transfer vector encoding CD40 ligand-treated animals as effectors and cisplatin-treated Lewis lung carcinoma cells as targets.

Results: Cisplatin induced heightened expression of Fas receptor on Lewis lung carcinoma cells in vitro and in vivo and enhanced apoptosis in cells exposed to an agonistic anti-Fas antibody. In vivo, the combination of 1 dose of intraperitoneal cisplatin and intratumoral adenovirus gene transfer vector encoding CD40 ligand inhibited tumor growth and prolonged survival compared with adenovirus gene transfer vector encoding CD40 ligand alone, resulting in a higher cure rate. This effect was lost in Fas ligand-deficient mice. Splenocytes from adenovirus gene transfer vector encoding CD40 ligand-treated wild-type mice lysed cisplatin-treated Lewis lung carcinoma cells more efficiently than untreated Lewis lung carcinoma cells, an effect lost in splenocytes from Fas ligand-deficient mice.

Conclusion: Cisplatin augments the antitumor effect of a cytotoxic T-lymphocytemediated immunotherapy strategy, resulting in a higher cure rate than seen with immunotherapy alone. This effect is associated with the enhanced ability of cytotoxic T lymphocytes to lyse tumor cells that have been exposed to cisplatin through Fas/Fas ligand interactions.

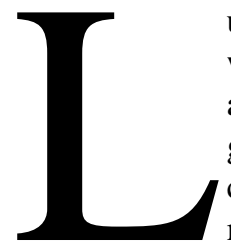

ung cancer is the leading cause of cancer mortality in men and women in the United States. " Despite the use of "conventional" antineoplastic therapies (chemotherapy, radiation therapy, and surgical resection), fewer than $15 \%$ of patients diagnosed with lung cancer will be cured of their disease. ${ }^{1}$ As most patients die of widely metastatic disease, there is clearly a need for novel systemic therapies. Active immunotherapy represents a systemic treatment strategy that may be 
potentially useful in many types of malignant disease, including lung cancer. By a variety of mechanisms, these strategies generally focus on enhancing the generation of cytotoxic T lymphocytes (CTLs), which circulate throughout the body and specifically induce tumor cell lysis. ${ }^{2-4}$

Our laboratory has focused on the development of novel active immunotherapy strategies utilizing in vivo adenovirus (Ad)-mediated gene transfer to induce CTL responses. In this context, a representative approach has been the direct transduction of established tumors with an Ad vector encoding the CD40 ligand cDNA (AdCD40L), which results in tumor-specific CTL generation, regression of established tumors, and long-term protection against tumor rechallenge. ${ }^{5}$ Although the antitumor effects of AdCD40L are demonstrable in multiple murine tumor models, they are less pronounced in the poorly immunogenic Lewis lung carcinoma (LLC) model, resulting in a negligible long-term cure rate. ${ }^{5}$

Cisplatin is a platinum-based, chemotherapeutic agent that has formed the backbone of systemic therapeutic regimens for both small cell and non-small cell lung cancer over the last 2 decades. ${ }^{6-8}$ In addition to its cytotoxic effects, cisplatin has been shown to enhance expression of the cell death receptor, Fas, in multiple human tumor cell lines in vitro, including lung cancer. ${ }^{9-12}$ Finally, many human lung cancers are poorly immunogenic and do not express significant levels of Fas. ${ }^{13-15}$ Given this information, as well as the limited antitumor effect of AdCD40L in the poorly immunogenic LLC model, we hypothesized that cisplatin may enhance the antitumor immunity evoked by AdCD40L in this model and that this enhancement would be due to heightened ability of tumor-specific CTLs to induce killing of LLC. In this regard, the data demonstrate: (1) exposure of LLC cells to cisplatin increases Fas expression in vitro and the up-regulated Fas serves as a functional cell death receptor; (2) 1 dose of intraperitoneal cisplatin induces intense Fas expression by established LLC tumors in syngeneic mice, an effect that lasts for at least 3 days; (3) the addition of 1 dose of intraperitoneal cisplatin to the administration of AdCD40L into established LLC tumors results in enhanced tumor regression and long-term survival when compared with the vector alone; (4) splenocytes from AdCD40Ltreated mice lyse cisplatin-exposed LLC cells more efficiently than naïve LLC cells; and (5) these enhanced antitumor effects of cisplatin are completely abrogated in Fas ligand-deficient mice.

\section{Materials and Methods \\ Mice}

Six- to eight-week-old female wild-type C57BL/6 $\left(\mathrm{H}-2^{\mathrm{d}}\right)$ and Fas ligand-deficient C57BL/6 (B6Smn.CH3-Tnfsf6 ${ }^{\text {gld }}$ ) mice were obtained from Jackson Laboratories (Bar Harbor, Me). The mice were housed under specific pathogen-free conditions and treated according to the National Institutes of Health guidelines. The Fas ligand $-/-$ phenotype was confirmed by flow cytometric analysis of splenocytes (not shown). All animal procedures were approved by the Institutional Animal Care and Use Committee.

\section{Cell Culture}

Lewis lung carcinoma $\left(\mathrm{H}-2^{\mathrm{d}}\right.$; a murine lung carcinoma) and B16.F10 (H-2 ${ }^{\mathrm{d}}$; a murine melanoma) are both syngeneic to C57BL/6 $\left(\mathrm{H}-2^{\mathrm{d}}\right)$ mice. The cell lines were obtained from American Type Culture Collection (ATCC; Manassas, Va) and maintained in complete Dulbecco's modified Eagle's medium (DMEM; $10 \%$ fetal bovine serum, $100 \mu \mathrm{g} / \mathrm{mL}$ streptomycin, and $100 \mathrm{U} / \mathrm{mL}$ penicillin) at $5 \% \mathrm{CO}_{2}$ and $37^{\circ} \mathrm{C}$.

\section{Adenovirus Vectors}

All gene transfer vectors used in this study are replication-deficient, E1-, E3- vectors based on the adenovirus serotype 5 (Ad5) genome. AdCD40L contains an expression cassette with the murine CD40L cDNA driven by the cytomegalovirus (CMV) earlyimmediate promoter/enhancer. ${ }^{5}$ AdNull, a control vector, is similar but contains no transgene. ${ }^{16}$ Ad vectors were propagated in human embryonic kidney cells (293 cells; ATCC) and purified through 2 cesium chloride gradient ultracentrifugations as previously described. ${ }^{17,18}$ The viral particle concentration was determined by ultraviolet absorbance at $260 \mathrm{~nm} .{ }^{19}$

\section{Quantification of Fas Receptor Expression and Fas- mediated Apoptosis in Vitro}

To demonstrate the ability of cisplatin to up-regulate Fas receptor in LLC cells in vitro, nearly confluent LLC cells were exposed to either $2.5 \mu \mathrm{g} / \mathrm{mL}$ or $5.0 \mu \mathrm{g} / \mathrm{mL}$ of cisplatin (Sigma Chemical Co, St Louis, Mo) for 3 hours in complete DMEM at $37^{\circ} \mathrm{C}$. The cells were washed 3 times with phosphate-buffered saline (PBS) and cultured in complete DMEM for an additional 36 hours. Shamtreated LLC cells served as controls. The cells were then stained with a phycoerthythrin $(\mathrm{PE})$-conjugated rat anti-murine Fas monoclonal antibody (mAb; Pharmingen, San Diego, Calif) or the appropriate isotype-matched, PE-conjugated control mAb (Pharmingen) and subjected to flow cytometric analysis (FACSCalibur; Becton Dickinson, San Jose, Calif).

To assess the functional activity of the up-regulated Fas receptor, LLC cells were cultured in complete DMEM containing 5 $\mu \mathrm{g} / \mathrm{mL}$ of cisplatin for 3 hours. The cells were then washed 3 times with PBS and cultured in complete DMEM at $37^{\circ} \mathrm{C}$. After 24 hours, $1 \mu \mathrm{g} / \mathrm{mL}$ of an agonistic rat anti-mouse Fas mAb (Jo2; Pharmingen) was added to the media and incubated for an additional 24 hours at $37^{\circ} \mathrm{C}$. The cells were washed 3 times with PBS and stained with fluoroisothiocyanate (FITC)-conjugated annexin V (R\&D Systems, Minneapolis, Minn) and TO-PRO-3 iodide (Molecular Probes, Eugene, Ore) for 15 minutes at $23^{\circ} \mathrm{C}$. The cells were then subjected to flow cytometric analysis (FACSCalibur).

\section{Immunofluorescence}

To assess the ability of cisplatin to enhance Fas expression on established tumors in vivo, $5 \times 10^{5}$ LLC cells were injected subcutaneously in the right flanks of wild-type C57BL/6 mice. When the tumors were approximately 45 to $50 \mathrm{~mm}^{2}$ in size (day 8), $7 \mathrm{mg} / \mathrm{kg}$ of cisplatin was administered as a single intraperitoneal dose. The animals were sacrificed and the tumors harvested 24 and 
72 hours after cisplatin injection. Sham-treated tumor-bearing mice served as controls. Tumors were snap-frozen in liquid nitrogen, cut into $10-\mu \mathrm{m}$ sections, and fixed in cold acetone. After blocking nonspecific staining with normal goat serum, the samples were treated with rabbit anti-mouse Fas receptor mAb (Santa Cruz Biotech, Santa Cruz, Calif) in 5\% goat serum for 2 hours. The samples were then incubated with an FITC-labeled goat anti-rabbit IgG (Santa Cruz Biotech) for 1 hour in 5\% normal goat serum, followed by 5 minutes in 4',6-diamidino-2-phenylindole $(1 \mu \mathrm{g} /$ $\mathrm{mL}$; Molecular Probes) in $0.1 \%$ Triton X-100 to stain nuclei (to verify the presence of intact cells on the slides). The sections were then assessed using fluorescence microscopy.

\section{Ability of Tumor-specific CTLs to Lyse LLC Cells}

To demonstrate the effect of cisplatin on the ability of AdCD40Linduced CTLs to lyse LLC cells, LLC tumors were initiated in the flanks of wild-type C57BL/6 mice as described above. When the tumors reached approximately 45 to $50 \mathrm{~mm}^{2}$ in size (day 8), AdCD40L or AdNull was administered intratumorally in $100 \mu \mathrm{L}$ PBS $\left(5 \times 10^{10}\right.$ particles $)$. Untreated animals served as further controls. Ten days after vector administration, spleens were harvested and minced and the red blood cells lysed using ACK buffer (Biosource International, Camarillo, Calif). Splenocytes from 3 animals in each group were pooled, and $8 \times 10^{6}$ splenocytes were restimulated with $2 \times 10^{6}$ mitomycin C-treated LLC cells (100 $\mu \mathrm{g} / \mathrm{mL}$ mitomycin $\mathrm{C}$ for 90 minutes; Sigma) for 5 days $(4 \mathrm{~mL}$ of complete RPMI-1640). After restimulation, viable effector cells (splenocytes) at varying effector-to-target ratios $(6: 1,20: 1,100: 1)$ were analyzed in a standard ${ }^{51} \mathrm{Cr}$-release assay for their ability to lyse ${ }^{51} \mathrm{Cr}$-labeled target cells during a 6-hour incubation at $37^{\circ} \mathrm{C}$. Target cells included untreated LLC cells, cisplatin-treated LLC cells, and untreated syngeneic control B16.F10 cells $\left(2 \times 10^{4} / 200\right.$ $\mu \mathrm{L} /$ well) The percentage of specific ${ }^{51} \mathrm{Cr}$-release was calculated as ([experimental release - spontaneous release]/[maximal release spontaneous release]) $\times 100$. To establish the role of Fas/Fas ligand interactions on the target cell killing ability of AdCD40Linduced CTLs, the same experiment was conducted using splenocytes obtained from tumor-bearing, Fas ligand-deficient mice (B6Smn.CH3-Tnfsf6 ${ }^{\text {gld }}$ ) after vector treatment.

\section{Evaluation of the Effect of Cisplatin Administration on the Antitumor Properties of AdCD40L in Vivo} LLC flank tumors were initiated as described above in wild-type C57BL/6 mice. When the tumors reached approximately 45 to 50 $\mathrm{mm}^{2}$ in size (day 8), AdCD40L or AdNull was administered intratumorally in $100 \mu \mathrm{L}$ PBS $\left(5 \times 10^{10}\right.$ particles $)$. Cisplatin $(7$ $\mathrm{mg} / \mathrm{kg}$ ) was administered as a single intraperitoneal dose concomitantly with vector injection. The size of the flank tumor was assessed in situ every 2 to 3 days by measuring the largest perpendicular diameters using microcalipers and recorded as an average tumor area $\left(\mathrm{mm}^{2}\right)$. When the animals appeared moribund or the tumor growth exceeded $15 \mathrm{~mm}$ in the largest diameter, the mice were killed, and this time point was defined as death for survival analysis. Untreated animals served as further controls. To assess the role of Fas/Fas ligand interactions in the effect of cisplatin on tumor immunity, the same experiment was conducted in Fas ligand-deficient mice (B6Smn.CH3-Tnfsf6 ${ }^{\mathrm{gld}}$ ).

\section{Statistical Analysis}

All data are reported as mean \pm standard error. Statistical significance between the means was determined using the unpaired, 2-tailed Student $t$ test. Survival evaluation was performed using the Kaplan-Meier analysis ( $P$ value determined by log-rank test).

\section{Results}

\section{Exposure of Lewis Lung Carcinoma to Cisplatin Increases Expression of Fas Receptor in Vitro and in Vivo}

The effect of cisplatin on the surface expression of Fas receptor was evaluated by exposing LLC cells to 2 different doses of cisplatin for 3 hours in culture and evaluating the cells using flow cytometry. LLC cells exposed to $2 \mu \mathrm{g} / \mathrm{mL}$ of cisplatin displayed heightened expression of Fas receptor compared with cisplatin-treated cells stained with an isotype-matched control mAb (not shown) or untreated cells (Figure 1, A). When the dose of cisplatin was increased to 5 $\mu \mathrm{g} / \mathrm{mL}$, only a minimal increase was demonstrated over the lower dose (Figure 1, B). To determine if this up-regulation could be demonstrated in vivo, LLC tumors were initiated in the flanks of syngeneic, wild-type mice and 1 intraperitoneal dose of cisplatin $(7 \mathrm{mg} / \mathrm{kg})$ was administered after the tumors were established. Tumors were harvested 24 and 72 hours after cisplatin dosing and evaluated for Fas expression using immunofluorescence. Although sham-treated tumors expressed negligible Fas receptor (Figure 2, $A$ and $B$ ), tumors from animals treated with cisplatin uniformly expressed Fas 24 hours after dosing (Figure 2, $C$ and $D$ ), an effect that was preserved for at least 72 hours (Figure 2, $E$ and $F$ ). No expression was detected in any tumors stained with the isotype-matched control Ab (not shown).

\section{Up-regulated Fas on LLC Cells Is a Functional Receptor for Apoptosis}

Because cisplatin enhances Fas expression both in vitro and in vivo, experiments were conducted to determine if this up-regulated receptor would initiate apoptosis of the LLC cells upon ligation. LLC cells were exposed to $5 \mu \mathrm{g} / \mathrm{mL}$ cisplatin in vitro for 3 hours; 24 hours later, apoptosis was assessed using annexin staining and flow cytometry. Although sham-treated LLC cells in culture contained some dead cells (Figure 3, A, upper 2 quadrants), cells undergoing apoptosis were not detected (lower, right quadrant). Exposure of LLC cells to the agonistic anti-Fas mAb (Jo2) had no effect on apoptosis (Figure 3, B). Cisplatin exposure alone enhanced apoptosis in LLC cells (Figure 3,C), but this effect was markedly potentiated by the addition of the agonistic anti-Fas mAb (Figure 3,D).

\section{CTLs from AdCD40L-treated, Tumor-bearing Mice Lyse Cisplatin-treated LLC Cells More Efficiently Than Untreated LLC Cells}

To determine whether the tumor-specific CTLs induced by AdCD40L will lyse LLC cells more efficiently if they have 


\section{A. $2 \mu \mathrm{g} / \mathrm{ml}$}

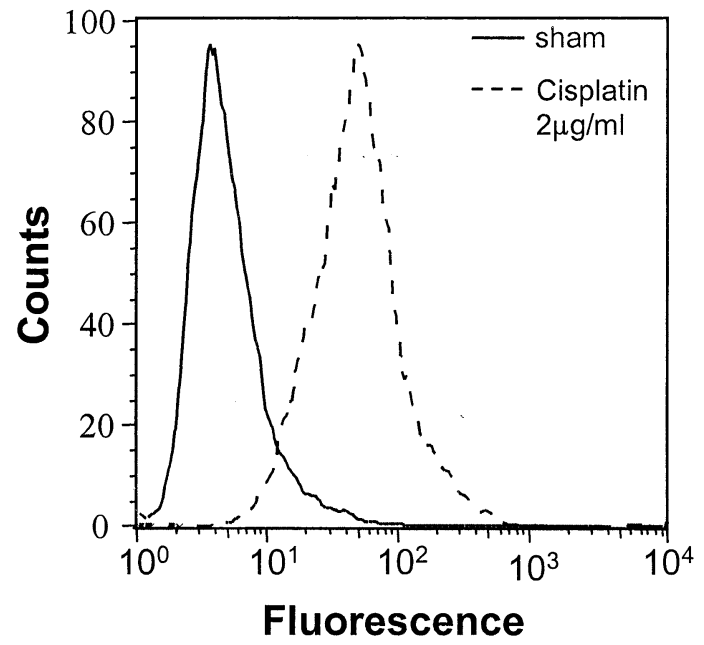

\section{B. $5 \mu \mathrm{g} / \mathrm{ml}$}

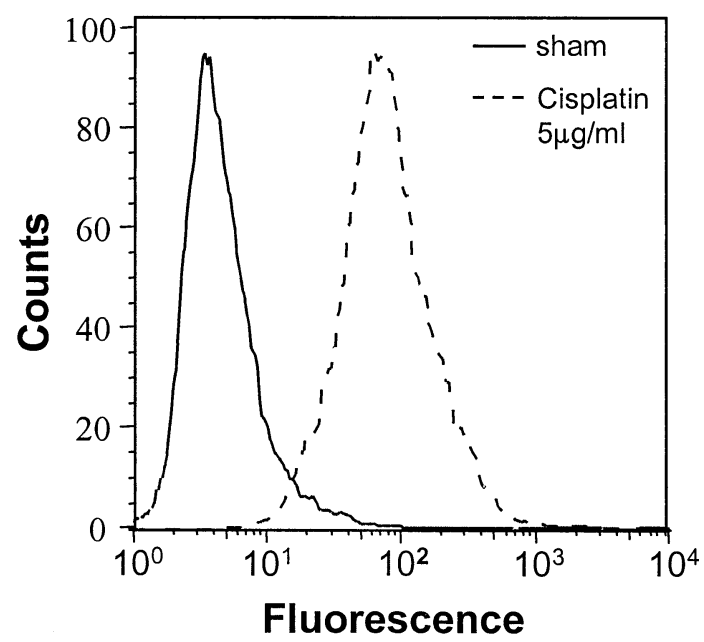

Figure 1. Exposure of LLC cells to cisplatin in vitro increases expression of Fas receptor. LLC cells were exposed to cisplatin at 2 different doses for $\mathbf{3}$ hours; 36 hours after exposure, the cells were stained with either a PE-conjugated rat anti-mouse Fas receptor $\mathrm{mAb}$ or an appropriate isotype-matched control $\mathrm{mAb}$ and subjected to flow cytometry. Sham-treated cells served as controls. The results are representative of 3 independent experiments. A, LLC cells treated with $2.0 \mu \mathrm{g} / \mathrm{mL}$ of cisplatin. B, LLC cells treated with $5.0 \mu \mathrm{g} / \mathrm{mL}$ of cisplatin. No staining was seen in the isotype-matched control groups (not shown).

been exposed to cisplatin, splenocytes from wild-type mice receiving an intratumoral injection of AdCD40L were isolated, restimulated in vitro, and evaluated in standard cytotoxicity assays. During a 6-hour incubation, splenocytes from AdCD40L-treated mice lysed LLC cells more effec- tively than untreated LLC cells (Figure 4, $A$ and $B$ ). However, these splenocytes were unable to lyse the syngeneic, control cell line, B16.F10 (Figure 4, C). Similarly, there was no lysis noted when splenocytes from either AdNull-treated or untreated tumor-bearing mice were incubated with any target cell (Figure 4, $A, B$, and $C$ ).

To evaluate the role of Fas/Fas ligand interactions in the ability of these tumor-specific CTLs to lyse target cells, the same experiment was repeated in Fas ligand-deficient mice. Splenocytes from these mice were unable to lyse either LLC cells or B16.F10 cells, regardless of cisplatin treatment (Figure $4, D, E$, and $F$ ).

\section{Addition of Cisplatin to a CTL-inducing Immunotherapy Strategy in Vivo Enhances Tumor Regression and Prolongs Survival}

To evaluate whether the addition of cisplatin enhances the antitumor effect of AdCD40L in vivo, LLC tumor-bearing wild-type C57BL/6 mice were given intratumoral AdCD40L as well as intraperitoneal cisplatin (1 dose of each), and tumor size was assessed over time. Tumor regression was more pronounced in the animals that received cisplatin plus AdCD40L (Figure 5, A) compared with that seen in the mice receiving AdCD40L alone $(P=.04)$, AdNull alone $(P=.002)$, cisplatin alone $(P=.00009)$, AdNull plus cisplatin $(P=.0002)$, or no therapy $(P=$ .000003). To establish the role of Fas/Fas ligand interactions in this antitumor effect, the same experiment was repeated in Fas ligand-deficient mice. Strikingly, the ability of cisplatin to enhance the antitumor effect of AdCD40L was abrogated in these transgenic mice (Figure $5, B ; P=.2$; AdCD40L plus cisplatin versus AdCD40L alone).

Because intratumoral AdCD40L alone results in a minimal cure rate when administered to established LLC tumors in syngeneic mice, animals were followed with survival as the end point. In wild-type C57BL/6 mice, significantly more animals receiving the combination of AdCD40L and cisplatin were cured of their tumors than those receiving the vector alone (Figure $6, A ; 43 \%$ versus $14 \% ; P=.03$ ). In contrast, no Fas ligand-deficient animals were cured of their tumors, regardless of treatment (Figure 6,B).

\section{Discussion}

Although innate antitumor immune responses exist, adaptive, specific tumor immunity is generally thought to be a cellular process, mediated by CD8 + CTLs as the immune effector cells. ${ }^{2-4}$ Activated CTLs are thought to induce cell death in target tumor cells mainly by the perforin/granzyme pathway, a process that requires direct cellular contact and target cell recognition via major histocompatibility complex class I. ${ }^{3,4,20}$ However, activated CTLs also express Fas ligand, which may bind to and induce apoptosis in tumor cell targets if the tumor expresses the Fas receptor. ${ }^{3,4,20}$ Lack of Fas expression by tumors may represent 1 mecha- 

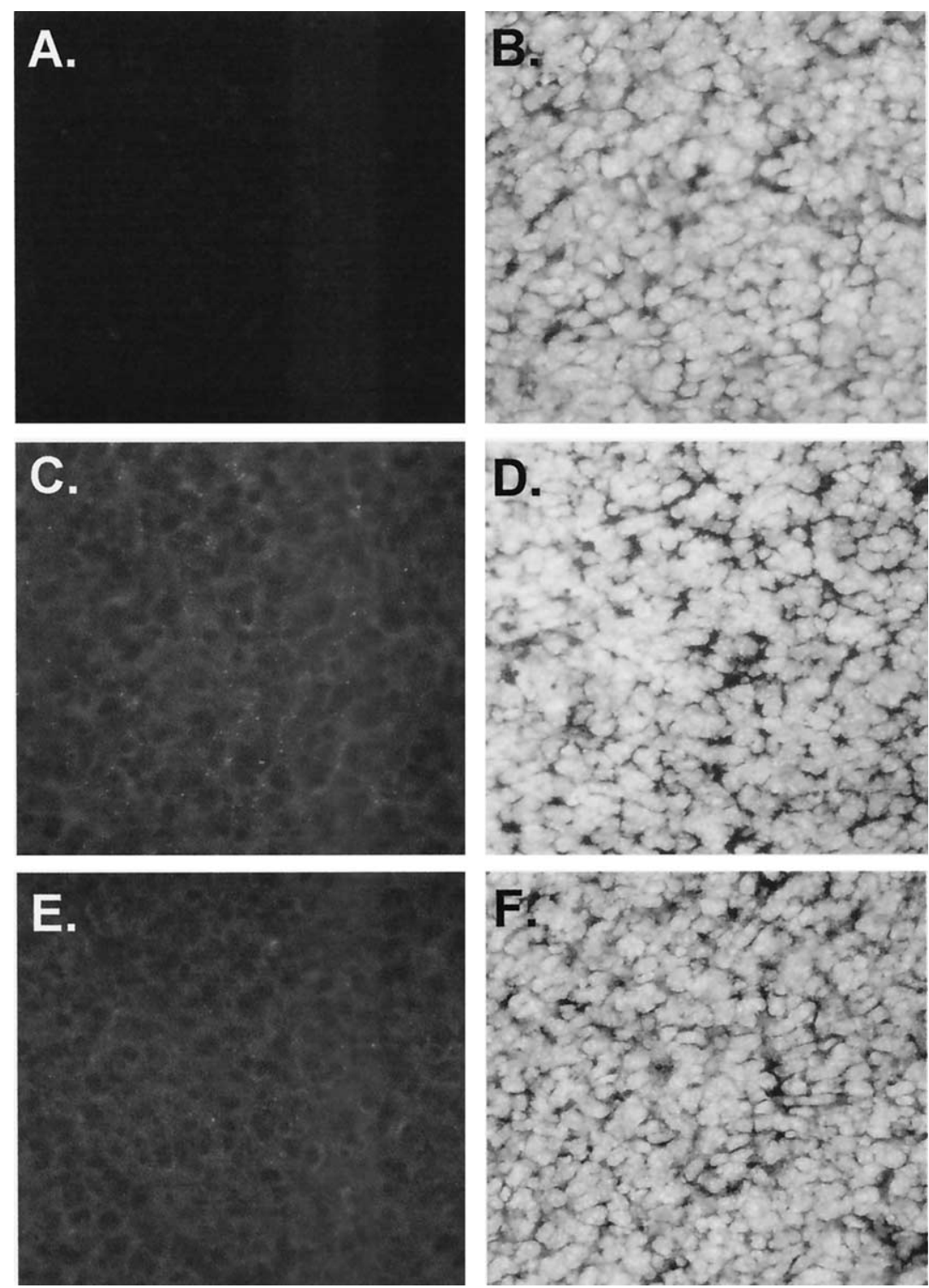

Figure 2. Intraperitoneal administration of cisplatin increases expression of Fas receptor in established LLC flank tumors. Tumors were initiated in syngeneic, wild-type mice and cisplatin was administered as a single intraperitoneal dose 8 days later; 24 and 72 hours after cisplatin treatment $(7 \mathrm{mg} / \mathrm{kg})$, the tumors were harvested, snap-frozen, and evaluated using immunofluorescence. Controls consisted of sham-treated mice as well as isotype-matched control $\mathrm{mAb}$ used in the staining protocol. Green fluorescence indicates Fas expression. A, Sham treatment. B, Nuclear staining from section in A. C, Twenty-four hours after cisplatin dosing. D, Nuclear staining from section in C. E, Seventy-two hours after cisplatin dosing. F, Nuclear staining from section in $E$. No green fluorescence was demonstrable in any sections exposed to the isotype-matched control $\mathrm{mAb}$.

nism by which tumors can evade CTL-mediated immune responses. For example, Fas receptor expression is absent or reduced in human lung cancers, affecting the susceptibility of these tumors to Fas-mediated apoptosis. ${ }^{14}$

Given the activity of cisplatin-based chemotherapeutic regimens in small cell and non-small cell lung cancer and the observation that cisplatin increases Fas receptor expression on multiple tumor cell lines in vitro, the present study was designed to determine whether cisplatin will enhance the antitumor effect of a CTL-inducing immunotherapy strategy based on its ability to up-regulate Fas expression on tumors. The data show that cisplatin markedly increases Fas expression in a poorly immunogenic, syngeneic murine lung cancer in vitro and in vivo and that this up-regulated Fas is a functional apoptosis receptor. In addition, tumorspecific CTLs are able to lyse cisplatin-treated LLC cells 


\section{A. Sham}

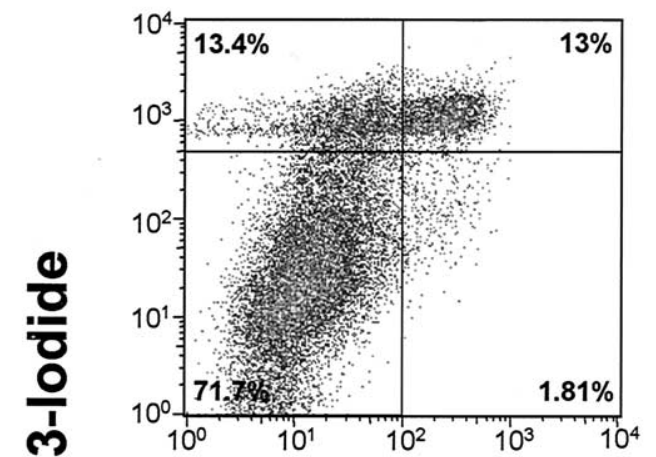

\section{Cisplatin}

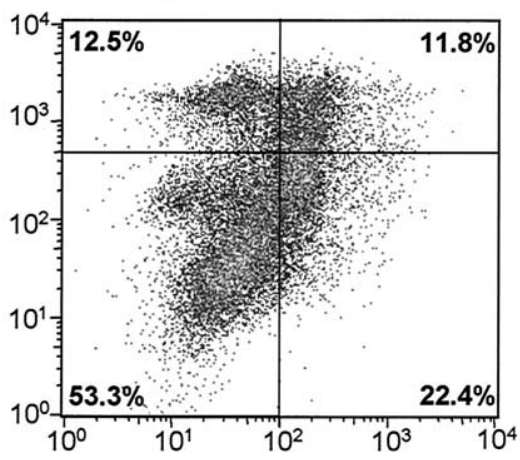

B. Jo2
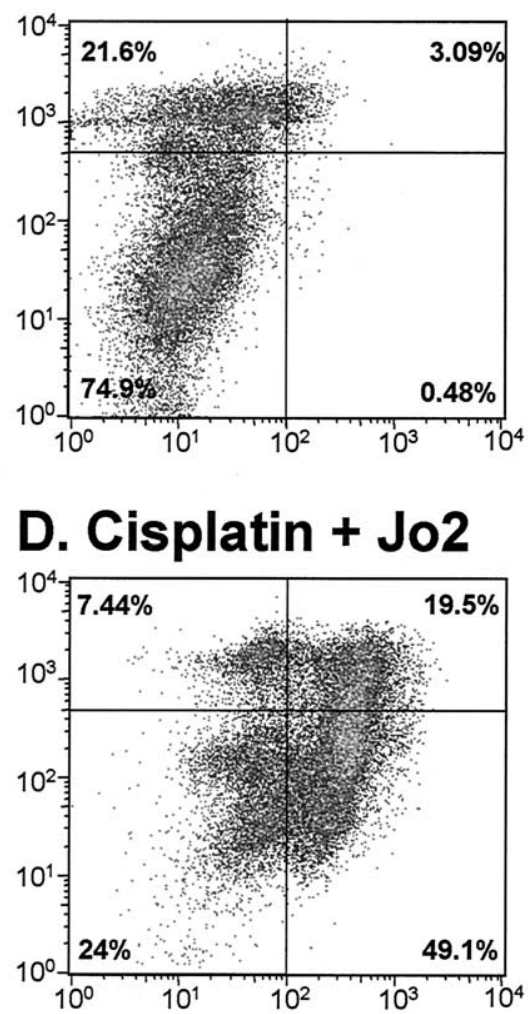

\section{FITC-Annexin V}

more effectively than untreated LLC cells, which translates into more pronounced tumor regression and better cure rate when cisplatin is combined with an active immunotherapy strategy in vivo.

Cisplatin Enhances Tumor Cell Killing by AdCD40Lgenerated CTLs via Fas/Fas Ligand Interactions

The susceptibility of tumors to active immunotherapy strategies is dependent on both the generation of CTLs and the ability of CTLs, once activated, to induce apoptosis in the tumor cells. ${ }^{2-4}$ LLC is a murine tumor refractory to active immunotherapy compared with other syngeneic murine tumors, exemplified by the consistent lack of long-term cures in mice bearing LLC tumors treated with immunotherapies. ${ }^{5,21}$ As a result, LLC represents an appropriate model in which to evaluate the effect of cisplatin.
The present study suggests that cisplatin augments the ability of tumor-specific CTLs to induce Fas ligand-mediated apoptosis of LLC cells, resulting in more pronounced tumor regression in a flank tumor model. This is supported by several observations. First, LLC cells express little Fas receptor, which is intensely up-regulated by the addition of cisplatin in vitro and in vivo. Although the in vitro concentrations of cisplatin probably do not represent in vivo physiologic concentrations of this agent, the in vivo data clearly show that Fas receptor is up-regulated at a dose that does not induce toxicity or tumor regression (a "subtherapeutic" dose). Second, CTLs from AdCD40L-treated wild-type mice lyse LLC cells more effectively if the LLC cells have been exposed to cisplatin. Finally, complete abrogation of this enhanced cell lysis occurs in Fas ligand-deficient mice. Interestingly, CTLs from Fas ligand-deficient mice pos- 

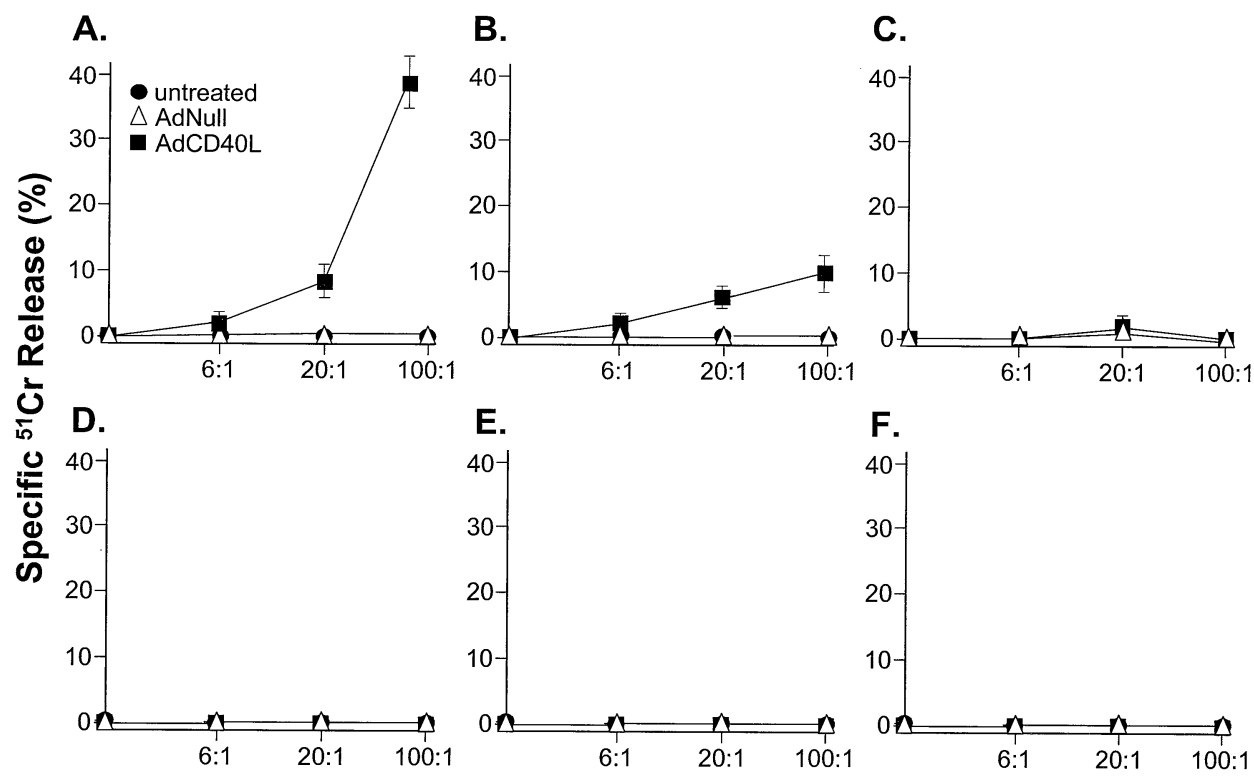

\title{
Effector to target ratio
}

\begin{abstract}
Figure 4. CTLs from tumor-bearing, wild-type C57BL/6 mice are more effective at lysing LLC cells exposed to cisplatin than untreated LLC cells, an effect abrogated in Fas ligand-deficient mice. Ten days following injection of either AdCD40L and AdNull $\left(5 \times 10^{10}\right.$ particles in $\left.100 \mu \mathrm{L}\right)$ into established LLC tumors, splenocytes were harvested, restimulated with mitomycin C-treated LLC cells for 5 days in vitro, and evaluated for cytotoxicity against target LLC cells or cisplatin-treated target LLC cells in a standard ${ }^{51} \mathrm{Cr}$ release assay. B16.F10 cells were used as a control target cell line. Untreated LLC tumor-bearing mice served as further controls. Data points represent the mean \pm SEM of 5 wells for each group. Shown is a representative experiment of 3 individual studies, all with similar results. A, Wild-type splenocytes, LLC target cells treated with cisplatin ( $5 \mu \mathrm{g} / \mathrm{mL})$. B, Wild-type splenocytes, untreated LLC target cells. C, Wild-type splenocytes, B16.F10 target cells. D, Fas ligand-deficient splenocytes, LLC target cells treated with cisplatin ( $5 \mu \mathrm{g} / \mathrm{mL}$ ). E, Fas ligand-deficient splenocytes, untreated LLC target cells. F, Fas ligand-deficient splenocytes, B16.F10 target cells.
\end{abstract}

sessed no ability to lyse LLC in any of the groups, implying that the major mechanism of apoptosis induction by CTLs generated via AdCD40L is the Fas/Fas ligand pathway, as opposed to the perforin/granzyme pathway. This finding is consistent with other published data showing CTLs can induce tumor cell apoptosis in the absence of perforin. ${ }^{22}$

Immunohistochemical analysis of human lung cancer has revealed Fas expression in only 30 to $50 \%$ of tumors. ${ }^{15}$ In addition, lung cancer patients with Fas-expressing tumors have been demonstrated to have significantly longer median survival than patients with Fas-negative tumors, ${ }^{14}$ and apoptosis induced by an agonistic anti-Fas antibody has been shown to correlate with the degree of Fas expression in human lung cancer cell lines. ${ }^{13}$ Finally, up-regulation of Fas receptor on human tumor cell lines has resulted in accentuated Fas-mediated cytotoxicity by allogeneic peripheral blood lymphocytes. ${ }^{9}$ These data, combined with the data in the present study, suggest that up-regulation of Fas receptor by cisplatin may be of benefit when used in conjunction with active immunotherapy in patients with lung cancer.

\section{Clinical Implications of Cisplatin Combined With AdCD40L}

Because lung cancer is associated with poor survival, with most patients dying of disseminated disease, active immunotherapy warrants investigation as a novel therapeutic strategy. However, tumor-associated antigens are poorly characterized and heterogenously expressed in human lung cancers, making antigen-specific vaccination strategies problematic. AdCD40L is a strategy that does not require prior knowledge of specific tumor antigens and, as such, may represent a valid approach for this disease. Because cisplatin is an established drug in the treatment of lung cancer, the finding that it also augments the antitumor effect of a CTL-mediated immunotherapy strategy is highly relevant for clinical application. A potential drawback of this approach is that the ability of chemotherapeutics to enhance Fas receptor expression in some cell lines requires the presence of wild-type $\mathrm{p} 53,{ }^{12}$ although human non-small cell lung cancer is associated with p53 mutations in approximately 50\% of cases. 


\section{A. Wildtype}

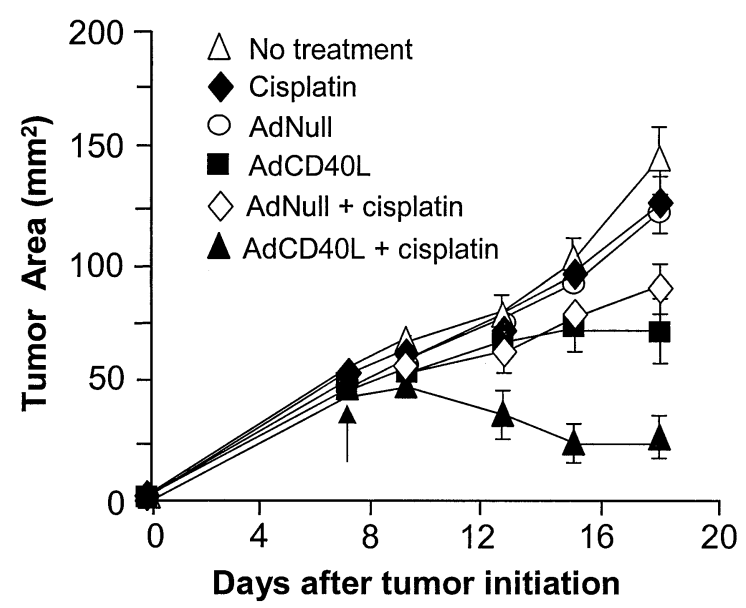

B. Fas ligand -/-

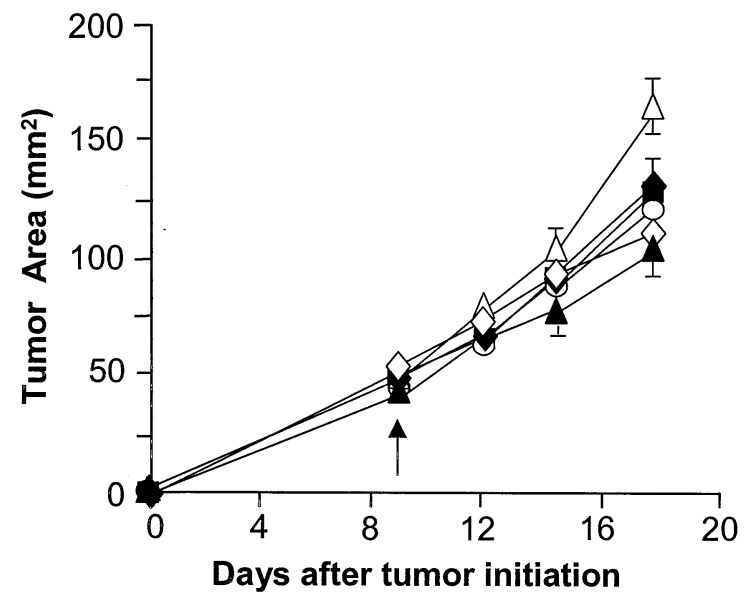

Figure 5. Cisplatin enhances the regression of established murine lung cancer induced by AdCD40L in wild-type mice, an effect abrogated in Fas ligand-deficient mice. Eight days after LLC flank tumor initiation, mice were randomized to 6 groups: AdCD40L plus cisplatin ( $n=7)$, AdCD40L alone ( $n=7)$, AdNull plus cisplatin ( $=6)$, AdNull alone $(n=7)$, cisplatin alone $(n=6)$, or untreated $(n=6)$. All vectors were given intratumorally $\left(5 \times 10^{10}\right.$ particles in $100 \mu \mathrm{L}$ ), and cisplatin was given as 1 concomitant, intraperitoneal dose $(7 \mathrm{mg} / \mathrm{kg})$. The tumor area was assessed in a blinded fashion at 2- to 3-day intervals. Data points represent the mean \pm SEM. A, LLC tumors in wild-type C57BL/6 mice. B, LLC tumors in Fas ligand-deficient mice (B6Smn.CH3-Tnfsf6 ${ }^{\text {gld }}$ ). The arrows indicate the time of tumor treatment.

Whether cisplatin will augment the antitumor activity of AdCD40L in tumors with mutated p53 remains to be determined.

We thank N. Mohamed for help in preparing this manuscript.

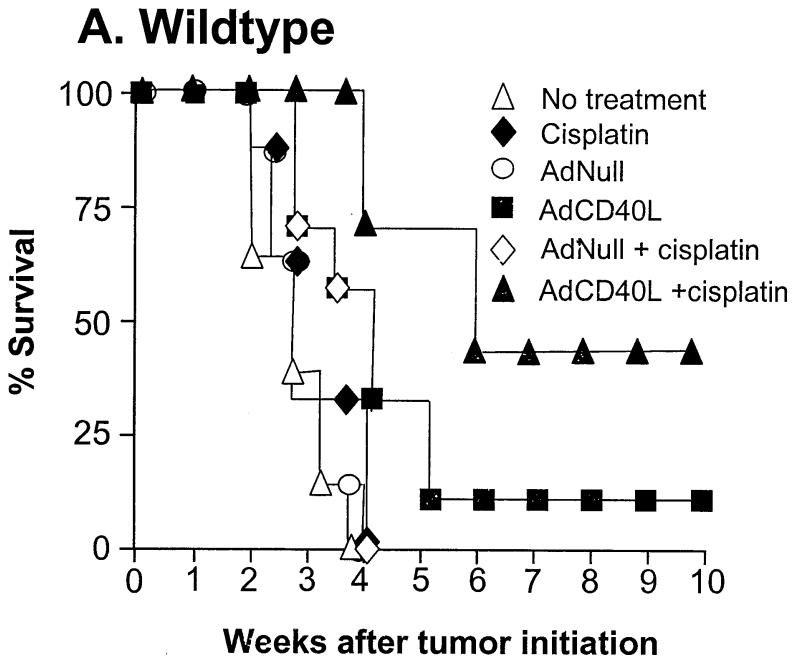

B. Fas ligand -/-

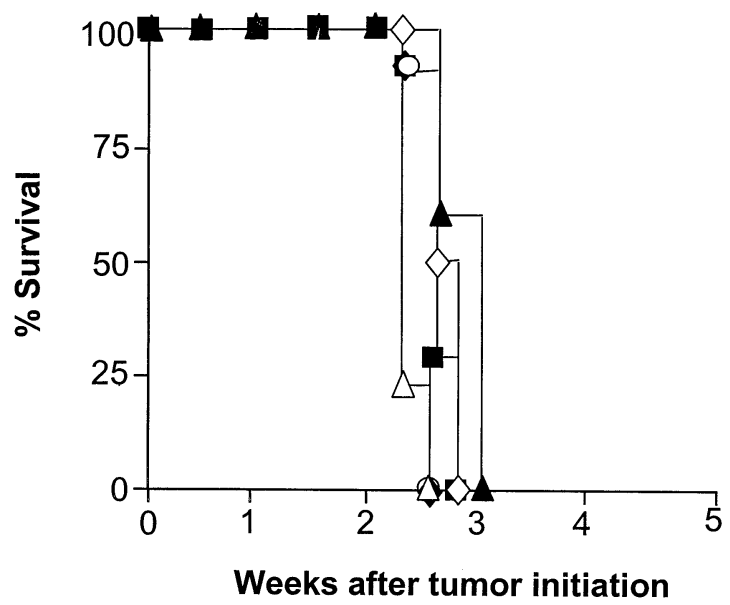

Figure 6. Cisplatin enhances the long-term cure rate of established murine lung cancer induced by AdCD40L in wild-type mice, an effect abrogated in Fas ligand-deficient mice. Eight days after LLC flank tumor initiation, mice were randomized to 6 groups: AdCD40L plus cisplatin ( $n=7)$, AdCD40L alone $(n=7)$, AdNull plus cisplatin $(n=6)$, AdNull alone $(n=7)$, cisplatin alone $(n=$ $6)$, or untreated $(n=6)$. All vectors were given intratumorally (5 $\times 10^{10}$ particles in $100 \mu \mathrm{L}$ ), and cisplatin was given as 1 concomitant, intraperitoneal dose $(7 \mathrm{mg} / \mathrm{kg})$. The mice were killed when the largest tumor diameter reached $15 \mathrm{~mm}$ or when the mice appeared moribund. A, LLC tumors in C57BL/6 mice. B, LLC tumors in Fas ligand-deficient mice (B6Smn.CH3-Tnfsf6 ${ }^{\text {gld }}$ ).

\section{References}

1. Greenlee RT, Murray T, Bolden S, Wingo PA. Cancer statistics, 2000. CA Cancer J Clin. 2000;50:7-33.

2. Gilboa E. The makings of a tumor rejection antigen. Immunity. 1999; 11:263-70.

3. Janeway CA, Travers P, Walport M, Capra JD. Immunobiology. The immune system in health and disease. 4th ed. New York: Garland Publishing; 1999. 
4. Abbas AK, Lichtman AH, Pober JS. Cellular and molecular immunology. 3rd ed. Philadelphia: W. B. Saunders; 1997.

5. Kikuchi T, Crystal RG. Anti-tumor immunity induced by in vivo adenovirus vector-mediated expression of CD40 ligand in tumor cells. Hum Gene Ther. 1999;10:1375-87.

6. Non-Small Cell Lung Cancer Collaborative Group. Chemotherapy in non-small cell lung cancer: a meta-analysis using updated data on individual patients from 52 randomized clinical trials. $\mathrm{Br}$ Med J. 1995;311:899-909.

7. Okuno SH, Jett JR. Small cell lung cancer: current therapy and promising new regimens. Oncologist. 2002;7:234-8.

8. Ettinger DS. Is there a preferred combination chemotherapy regimen for metastatic non-small cell lung cancer? Oncologist. 2002;7:226-33.

9. Micheau O, Solary E, Hammann A, Martin F, Dimanche-Boitrel MT. Sensitization of cancer cells treated with cytotoxic drugs to Fasmediated cytotoxity. J Natl Cancer Inst. 1997;89:783-9.

10. Stewart JH IV, Nguyen DM, Chen GA, Schrump DS. Induction of apoptosis in malignant pleural mesothelioma cells by activation of the Fas (Apo-1/CD95) death-signal pathway. J Thorac Cardiovasc Surg. 2002;123:295-302.

11. Matsuzaki I, Suzuki H, Kitamura M, Minamiya Y, Kawai H, Ogawa J. Cisplatin induces Fas expression in esophageal cancer cell lines and enhanced cytotoxicity in combination with LAK cells. Oncology. 2000;59:336-43.

12. Muller M, Wilder S, Bannasch D, et al. P53 activates the CD95 (Apo-1/Fas) gene in response to DNA damage by anticancer drugs. $J$ Exp Med. 1998;188:2033-45.

13. Kawasaki M, Kuwano K, Nakanishi Y, et al. Analysis of Fas and Fas ligand expression and function in lung cancer cell lines. Eur J Cancer. 2000;36:656-63.

14. Nambu Y, Hughes SJ, Rehemtulla A, Hamstra D, Orringer MB, Beer
DG. Lack of cell surface Fas/AOP-1 expression in pulmonary adenocarcinomas. J Clin Invest. 1998;101:1102-10.

15. Uramoto $\mathrm{H}$, Osaki $\mathrm{T}$, Inoue $\mathrm{M}$, et al. Fas expression in non-small lung cancer: its prognostic effect in completely resected stage III patients. Eur J Cancer. 1999;35:1462-65.

16. Hersh JR, Crystal RG, Bewig B. Modulation of gene expression after replication deficient, recombinant adenovirus-mediated gene transfer by the product of a second adenovirus vector. Gene Ther. 1995;2:12431.

17. Rosenfeld MA, Yoshimura K, Trapnell BC, et al. In vivo transfer of the human cystic fibrosis transmembrane conductance regulator gene to the airway epithelium. Cell. 1992;68:143-55.

18. Rosenfeld MA, Siegfried W, Yoshimura K, et al. Adenovirus-mediated transfer of a recombinant alpha 1-antitrypsin gene to the lung epithelium in vivo. Science. 1991;252:431-4.

19. Mittereder N, March KL, Trapnell BC. Evaluation of the concentration and bioactivity of adenovirus vectors for gene therapy. J Virol. 1996; 70:7498-509.

20. Kagi D, Lederman B, Burki K, Zinkernagel RM, Hengartner H. Molecular mechanisms of lymphocyte-mediated cytotoxicity and their role in immunological protection and pathogenesis in vivo. Аnnu Rev Immunol. 1996;14:207-32.

21. Fushimi T, Kojima A, Moore MA, Crystal RG. Macrophage inflammatory protein 3 alpha transgene attracts dendritic cells to established murine tumors and suppresses tumor growth. J Clin Invest. 2000;105: 1383-93.

22. Seki N, Brooks AD, Carter CRD, et al. Tumor-specific CTL kill murine renal cancer cells using both perforin and Fas ligand-mediated lysis in vitro, but cause tumor regression in vivo in the absence of perforin. J Immunol. 2002;168:3484-92. 